\title{
Andropause - state of the art 2015 and review of selected aspects
}

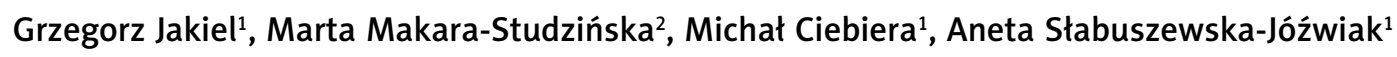 \\ ${ }_{1}^{1}$ Department of Obstetrics and Gynaecology, Centre of Postgraduate Medical Education, Warsaw, Poland \\ ${ }^{2}$ Department of Applied Psychology, II Faculty of Medicine with English Language Division, Medical University of Lublin, Lublin, Poland
}

\begin{abstract}
The term 'male menopause' was first used in 1944 to describe various complaints of ageing men which at least partially mirrored the climacteric symptoms in women. Continuous research resulted in the evolution of opinions about the nature of these complaints, from the clinical syndrome, diagnosed with the use of diseasetargeted questionnaires, to a well-defined clinical and biochemical syndrome. The pathophysiological causes - gonad ageing (with a compensatory rise in luteinizing hormone), age-related increase in serum sex hormonebinding globulin (SHBG) levels, the role of visceral adipose tissue as a place for aromatization of androgen to estrogen, and lower sensitivity of testosterone receptors - have been described. However, no consensus was reached as far as the definition, incidence, treatment, and especially testosterone therapy, are concerned. Our review presents the current standpoints, indicating the predictive role of late-onset hypogonadism (LOH) in evaluating male health as well as the current literature reports on the risks and benefits of using testosterone therapy.

Key words: late-onset hypogonadism, ageing male, andropause.
\end{abstract}

\section{Introduction}

Recently, a distinct rise in the incidence of psychological disturbances has been noted among middleaged men [1], thus indicating an urgent need for additional, in-depth studies to investigate that population. Andropausal men often have responsible professional functions and are at the peak of their careers but health and psychological problems hinder their goals and result in overall dissatisfaction, thus increasing emotional disturbances. The symptoms of emotional disorders among males at that point of their life include moodiness, irritability, nervousness, depression, aggravation, fatigability, poor concentration, deteriorating memory, worse stress management and stress-related coping techniques, new aversion to certain activities that usually were enjoyable, or prolonged mood disorders. In 2007, Sato et al., in their study on men from an andrology clinic, found depressive disorders in approximately $42 \%$ of their patients, emphasizing the need to screen for menopause-related mood disorders among men [2].

Anhedonia, often described by the affected individuals as inability to experience joy, is the basic symptom of depression. Also, the feeling of exhaustion and lack of energy, resulting in reduced psychomotor drive, what in turn leads to a slower thinking process and functional cognitive impairment, is another common sign of depression. According to numerous psychologists, andropause signifies the time when most men start to perceive their life as a whole, as a process rather than a sequence of individual actions. At that time, men conclude that their number of days is not unlimited, what is often accompanied by the feeling of unfulfillment. Men who struggle to accept the passing of time, may find it terrifying, what consequently might trigger rebellion and unorthodox behavior. These changes herald a potential breakthrough in a man's life, which leads to a more conscious life. Strong identification with the self-perceived attractiveness and strength makes it challenging to accept hormonal changes and the related phases of life. Emotional symptoms are accompanied by various somatic disorders. Thus, the unaccepted process of ageing begins.

Signs of male ageing often mirror menopausal complaints in women [3]. The deteriorated general and sexual condition in men was first identified in 1944 by Hellers and Meyers [4] who associated it with decreased testosterone levels and were the first to use the term 'male menopause'. Heinemann and Saad [5] reported that sudden and excessive sweating is not genderdependent and the Vermeulen group even claims that 'climacteric symptoms' affect both sexes and only vary in terms of incidence rates [6]. These assumptions validated the use of the term 'male menopause', or 'andropause'. Despite time and effort, emotional disorders resulting from decreased androgen levels are often treated as symptoms of depression or bipolar disease, and social awareness of the threatened well-being of men remains relatively low [7].

Corresponding author: Prof. Grzegorz Jakiel, I Department of Obstetrics and Gynaecology, Centre of Postgraduate Medical Education,

231 Czerniakowska St., 00-416 Warsaw, phone: +48 2258411 60, e-mail: kl.ginekologii@szpital-orlowskiego.pl

To cite this article: Jakiel G, Makara-Studzińska M, Ciebiera M, Słabuszewska-Jóźwiak A. Andropause - state of the art 2015 and review of selected

aspects. Prz Menopauzalny 2015; 14: 1-6.

Submitted: 6.03.2015; Accepted: 15.03.2015. 
The study by Turnover made it evident that the analogy between menopause and andropause may pertain to clinical manifestations but not the nature of the underlying hormonal changes. A decreased testosterone level - the main male hormone - is permanent rather than transient, and is approximately $1 \%$ per year $[8,9]$. A recent study by Belchetz et al. [10] determined the total testosterone drop to be $1.4 \%$ annually. The diagnosis of andropause was based on clinical symptoms evaluated with the use of more or less complex questionnaires that assessed subjective opinions of the patients. Wu et al. [11] generated the following results as far as the incidence of the major clinical symptoms is concerned:

- low libido - 91\%,

- lack of energy $-89 \%$,

- erectile dysfunction - 79\%,

- postprandial somnolence - $77 \%$,

- memory deterioration - 77\%,

- loss of pubic hair - 70\%,

- sadness and irritability -68\%,

- decreased endurance-66\%,

- loss of facial hair - 55\%,

- trouble at work - 51\%.

Two questionnaires for evaluating the intensity of the complaints have gained the largest popularity: the Ageing Males' Symptoms (AMS) scale of by Claudia Moore et al., and the Androgen Deficiency in Ageing Males (ADAM) scale of Morley et al. The former assessed 17 parameters of male well-being in three groups: psychological, somatic and sexual subscales [12], whereas the latter, often used in its simplified version, assumed a definite dominance of the sexual symptoms - in fact, their occurrence was the decisive factor to make the final diagnosis [13].

The ADAM questionnaire in extenso is included below:

1. Do you have a decrease in libido or sex drive?

2. Do you have a lack of energy?

3. Do you have a decrease in strength and/or endurance?

4. Have you lost height?

5. Have you noticed a decrease in your enjoyment of life?

6. Do you feel sad and/or grumpy?

7. Are your erections less strong?

8. Have you noticed a recent deterioration in your ability to play sports?

9. Do you fall asleep after dinner?

10. Has there been a recent deterioration in your performance at work? [13].

The questionnaires were perceived as independent diagnostic tools and their results deemed the patient eligible for testosterone therapy. The management did not aim to compensate the deficiency but to identify a group of patients with possible beneficial therapeutic effects after testosterone administration. In practice, hormonal therapy, especially American one, which is based on testosterone administration, was in fact the way to prevent ageing. Even then it was obvious that not all men, unlike women and the post-menopausal estrogen drop, have testosterone deficiency. The Massachusetts Male Ageing Study (MMAS) evaluated the same group of men on a long-term basis, and found that $21 \%$ of the investigated males aged 55-59, 26\% aged $60-69$, and $31 \%$ aged $70-86 \%$ did not meet the standards for testosterone levels [14]. A further study revealed the results to be significantly lower if the following two criteria were separated: presence of the clinical symptoms and testosterone levels below the norm.

The mean age of the sample was $47.3 \pm 12.5$ years. Approximately $24 \%$ of the subjects had total testosterone of $<300 \mathrm{ng} / \mathrm{dl}$, and $11 \%$ of subjects had free testosterone of $<5 \mathrm{ng} / \mathrm{dl}$. Prevalence of the symptoms was as follows: low libido (12\%), erectile dysfunction (16\%), osteoporosis/fracture (1\%), and two or more non-specific symptoms (20\%). Low testosterone levels were associated with symptoms, but many men with low testosterone levels were asymptomatic (e.g. men 50+ years, $47.6 \%)$. Crude prevalence of symptomatic androgen deficiency was $5.6 \%(95 \% \mathrm{Cl}: 3.6-8.6 \%)$, and was not significantly related to race and ethnicity. Prevalence was low in men < 70 years (3.1-7.0\%) and increased markedly with age to $18.4 \%$ among 70 -year-olds [15].

According to currently available studies, proper age-dependent reference ranges for testosterone do not exist and the vast majority of the published guidelines propose the cut-off value of $10 \mathrm{nmol} / \mathrm{l}$ [16]. The assumption behind testosterone therapy in men who presented only with clinical symptoms, without low testosterone levels in serum, was that, owing to a considerable individual variation in serum testosterone concentration, a result within the normal ranges does not exclude a significant decrease as compared to earlier results. Currently, in the era of evidence-based medicine, such interpretation does not stand the test of time.

In 2002, the International Society for Study of Ageing Male (ISSAM) published the first recommendations about diagnosis and treatment of late-onset hypogonadism (LOH), updated in 2005 and 2008. They assumed that the diagnosis of $\mathrm{LOH}$ ought to be made if low testosterone levels coincide with the above-mentioned clinical symptom. These guidelines abandon the practice of initiating treatment solely on the basis of only a positive result of the questionnaire study [17].

At present, Lunenfeld et al. suggested an improved version of these recommendations $[18,19]$. Their definition of $\mathrm{LOH}$, as well as interchangeably used testosterone deficiency syndrome (TDS), significantly expands the classic understanding of hypogonadism: $\mathrm{LOH}$, also referred to as age-related TDS, is a clinical and biochem- 
ical syndrome in men with advancing age (who have had normal pubertal development and normal male secondary characteristics) associated with low testosterone, age-related comorbidities, and deterioration in the general health status. Moreover, these authors claim that an elevated luteinizing hormone ( $\mathrm{LH}$ ) level with total testosterone below $15 \mathrm{nmol} / \mathrm{l}$ would also suggest $\mathrm{LOH}$. This notion is based on recently published studies, which in turn are the result of the European Male Ageing Study, indicating a marginal range of $\mathrm{LOH}$ incidence, defined as a decreased serum testosterone concentration [20]. In fact, $15 \mathrm{nmol} / \mathrm{l}$ is higher than the previously used population cut-off value for the total testosterone concentration in serum. These authors also go back to the notion of 'individual norm' of total testosterone. Since testosterone sensitivity may vary in different individuals, it has also been argued that the magnitude of the decrease in serum testosterone concentrations might be a better predictor of $\mathrm{LOH}$ than the actual concentrations of total testosterone and bioavailable testosterone. This observation is based on the results of Holm et al. The Turku Male Ageing Study started in 2000 and is still ongoing. It includes more than 15000 men above the age of 40 years. Current analyses indicate that several major questions about the importance of the ageing-related decline of testosterone production still remain unanswered. For example, a poor correlation between low testosterone levels and symptoms of late-onset hypogonadism raises the question as to whether the right methods are being used to assess the androgen status of ageing men. Interestingly, the Turku data show that, unlike some previous contentions, high testosterone levels in ageing men are not a risk factor for atherosclerosis [21].

A new large study published in the "New England Journal of Medicine" offers other perspectives. This multi-center study, the European Male Ageing Study (EMAS), recruited 3369 men between the ages of 40 and 79 years (mean 59.7 years), who were invited to undergo health assessment by questionnaire, physical and cognitive performance tests, and blood tests for biochemical and hormone measurements. Based on previous knowledge, 32 items from the EMAS questionnaires were considered as possible candidates for symptoms of androgen deficiency. However, only nine symptoms were confirmed as being related to the total or free testosterone levels, with significant differences between the symptomatic and the asymptomatic groups. These symptoms included three sexual manifestations (decreased frequency of morning erection, decreased frequency of sexual thoughts, and erectile dysfunction), three physical symptoms (inability to engage in vigorous activity, e.g. running, lifting heavy objects, or participating in strenuous sports; inability to walk more than $1 \mathrm{~km}$; and an inability to bend, kneel, or stoop), and three psychological symptoms (loss of en- ergy, sadness ['downheartedness' in the questionnaire], and fatigue). A range of other symptoms was not linked with the male menopause and could be ruled out, such as changes in the sleeping pattern, poor concentration, feeling worthless, nervousness and anxiety, as well as difficulty in getting up from a chair. Data also enabled the setting of the symptom thresholds: increased probability of the three sexual symptoms and limited physical vigor were already detectable with the following range of decreased testosterone levels: $8.0-13.0 \mathrm{nmol} / \mathrm{l}(2.3-$ $3.7 \mathrm{ng} / \mathrm{ml}$ ) for total testosterone, and 160-280 pmol// $(46-81 \mathrm{pg} / \mathrm{ml})$ for free testosterone.

However, only the three sexual symptoms had a syndromic association with decreased testosterone levels. An inverse relationship was observed between an increasing number of sexual symptoms and a decreasing testosterone level. The conclusion of the study was that $\mathrm{LOH}$ actually affects only $2 \%$ of men according to their criteria, defined by the presence of at least three sexual symptoms associated with a total testosterone level of $<11 \mathrm{nmol} / \mathrm{l}(3.2 \mathrm{ng} / \mathrm{ml})$ and a free testosterone level of $<220 \mathrm{pmol} / \mathrm{l}(64 \mathrm{pg} / \mathrm{ml})[15,16,21]$.

Ilpo Huhtaniemi terms these three clinical symptoms (decreased frequency of morning erection, decreased frequency of sexual thoughts, and erectile dysfunction correlated with a serum total testosterone level of < $11 \mathrm{nmol} / \mathrm{l}$ ) strict diagnostic criteria for $\mathrm{LOH}$, by the analogy to a notion (well-known among andrologists) of 'strict criteria' in relation to sperm morphology [16]. Notably, manifestations such as the 'strict criteria' many years ago tipped the scale for the positive result of the ADAM questionnaire. The EMAS supplied the basis for earlier historical, empirical observations. Even such strict criteria cannot be perceived as fully representative. Endocrine foundation of the erectile dysfunction is not only hypoandrogenism. Evaluation of thyroid stimulating hormone (TSH), $\mathrm{LH}$, and prolactin might be performed together with testosterone assessment. There is still no definite evidence of the role of estradiol, dihydrotestosterone $(\mathrm{DHT})$, dehydroepiandrosterone (DHEA), dehydroepiandrosterone sulfate (DHEA-S), cortisol, growth hormone $(\mathrm{GH})$, or insulin-like growth factor 1 (IGF-1) in erectile dysfunction (ED) [22].

The pathomechanism of $\mathrm{LOH}$ includes several possible paths resulting in decreased testosterone levels [23]:

- a decline in the function of Leydig cells and the pituitary-hypothalamic axis,

- an increase in the levels of sex hormone-binding globulin (SHBG), which occurs with advancing age and consequently leads to less free testosterone,

- the presence of visceral fat.

Visceral adipose tissue is the most metabolically active adipose tissue in human body and drains through the portal vein to the liver, the consequences of which 
lead to peripheral hyperinsulinemia and systemic insulin resistance. The underlying mechanisms responsible for the reduced testosterone levels in obese men are unknown. Possible mechanisms include a form of hypogonadotrophic hypogonadism, hyperoestrogenemia from peripheral conversion of testosterone which leads to a further reduction in serum and tissue testosterone concentrations, increased deposition of abdominal fat, and progressive hypogonadism [24]. Serum testosterone in a man with body mass index $(\mathrm{BMI})>30 \mathrm{~kg} / \mathrm{m}^{2}$ is, on average, $30 \%$ lower than that of a man with $\mathrm{BMI}$ $<25 \mathrm{~kg} / \mathrm{m}^{2}$, at any age, which is more than a purely age-dependent decrease between 40 and 80 years. $\mathrm{Cu}-$ riously, the decrease in testosterone secretion associated with obesity and chronic illness is not associated with a compensatory increase in gonadotropin levels, thus indicating a secondary central mechanism for the disturbance [20].

Visceral tissue also produces inflammatory cytokines and promotes endothelial dysfunction and vascular diseases [25].

A possible decline in the sensitivity of testosterone receptors, especially those in the central nervous system (CNS), may explain both the decrease in sexual desire in the ageing male and the need for a larger dose of testosterone when treating hypogonadism [23].

The above-mentioned phenomena may constitute the foundation for the debate, and the first two pathomechanisms are quite commonly accepted - gonad ageing compensated by increased pituitary activity stimulation via LH. The mechanism is as close as possible to menopause (low estrogens, high gonadotropin levels), despite the absence of the inability to produce estrogens, which is characteristic for climacteric women, in men gonad ageing occurs but its function is preserved, and additionally higher bonding to SHBG takes place, what results in lower androgenization.

Androgen aromatization in the visceral adipose tissue in obese men seems to be analogous to that in their sex counterparts. Also, the theory of lower receptor sensitivity for androgens is most promising. It allows to explain the coincidence of normal testosterone concentrations and clinical symptoms typical of andropause. Regardless, such pathomechanism does not account for the beneficial effect of androgen administration, which should have only limited value.

The model suggested by Wylie and Froggat allows to bring together the low rates of men with 'real' $\mathrm{LOH}$, i.e. defined according to the EAMS and earlier MMAS guidelines [15], with the common belief of clinicians that hypoandrogenism-related problems of ageing men remain underestimated.

A cross-sectional cohort study identified symptom specific threshold levels with androgen-induced prevalence of loss of libido or vigor increased when testosterone concentrations were below $15 \mathrm{nmol} / \mathrm{l}$, whereas depression and diabetes mellitus type 2 (also in nonobese men) were significantly higher in men with testosterone concentrations of $<10 \mathrm{nmol} / \mathrm{l}$ [26]. The problem of the cut-off value for serum testosterone remains to be resolved. In their latest recommendations, Lunenfeld et al. mention the value of $15 \mathrm{nmol} / \mathrm{l}[18,19]$.

In summary, there is general agreement that serum total testosterone levels of $8 \mathrm{nmol} / \mathrm{l}(231 \mathrm{ng} / \mathrm{dl})$ or free testosterone of $5180 \mathrm{pmol} / \mathrm{l}(52 \mathrm{pg} / \mathrm{ml})$ require substitution. A trial of testosterone is indicated for patients with total testosterone levels of between 8 and $12 \mathrm{nmol} / \mathrm{l}$, if there is evidence of clinical symptoms, and the free testosterone level is less than $225 \mathrm{pmol} / \mathrm{l}$ [27].

The fact that every laboratory has its own set of norms, whose literature background remains unknown, and that virtually all of them establish the lower norm at the level of $<300 \mathrm{ng} / \mathrm{ml}$, thus making it impossible to relate to the literature, constitutes an additional problem for Polish clinicians. Regardless, large population studies like MMAS and EAMS also used a lower cutoff value, and additionally the values were different in these two cases.

\section{Late-onset hypogonadism treatment}

As far as LOH treatment is concerned, it is important to emphasize that, according to the EMAS data, it is possible to state almost categorically that if a man does not have sexual symptoms he does not have $\mathrm{LOH}$ [20]. On the other hand, a significant number of men seeking medical help are reluctant to discuss the topic of sexual dysfunctions with their family doctor. Therefore, a medical interview with a low testosterone level patient ought to be very detailed and, as such, may present a challenge for the physician. It seems that, as in case of women and menopause, the first-line treatment consists in lifestyle changes.

Recent EMAS data show that weight gain or loss have a clear inverse correlation with circulating testosterone and vice versa. Testosterone treatment has important effects on body composition, resulting in a significant increase in lean body mass, and a significant decrease in fat mass without a change in body weight $[28,29]$. Moreover, the risk of experiencing adverse effects associated with testosterone treatment was reported to increase in obese subjects [30], therefore weight loss using testosterone therapy should be conducted with care and attention. Similar caution should be advised when suggesting a program of intense physical exercises as it may have a detrimental effect on the lipid profile in men, just like testosterone therapy [31].

Because $\mathrm{LOH}$ symptoms are similar to those of hypogonadism in young men, testosterone replacement therapy has become a popular treatment option. Improvement of symptoms should be assessed after a few months of treatment. If no benefits are reported 
by the patient, treatment should be terminated and other causes of symptoms should be investigated. If the treatment is effective, the patients should be monitored with regular checking of their hematocrit, hemoglobin and prostate specific antigen (PSA) levels and by doing digital rectal examination every 3-6 months [32]. Since the possible development of an adverse event during treatment, especially elevated hematocrit or prostate carcinoma, requires rapid discontinuation of testosterone substitution, short-acting preparations may be preferred over long-acting depot preparations in the initial treatment of patients with $\mathrm{LOH}$ [33].

Currently, absolute contraindications to testosterone therapy include prostate and breast cancer. Relative contraindications are serum PSA level of $>4 \mathrm{ng} / \mathrm{ml}$ (or $3 \mathrm{ng} / \mathrm{ml}$ in men with an increased risk of prostate cancer, hematocrit $>50 \%$, severe lower urinary tract symptoms caused by benign prostatic hypertrophy (as defined by an International Prostate Symptom Score [IPSS] higher than 19), untreated or poorly controlled congestive heart failure, and untreated sleep apnea [34]. A review of the literature shows that testosterone treatments have not been proven to be beneficial with respect to cardiovascular disease, nor have they definitely shown specific adverse cardiovascular effects [35].

We still lack randomized, placebo-controlled studies on testosterone replacement in men aged $\geq 60$ years. The existing trials have low numbers of subjects and are often of insufficient duration [36]. At the moment, a controlled-placebo study in a group of 800 men is being carried out in the USA, and the results are anticipated in 2015.

Currently, the testosterone therapy remains the topic of a much heated debate and the arguments are often radical, e.g. Handelsmann is of the opinion that the reasons for testosterone therapy are often non-medical, and that the marketing of testosterone treatments has been aggressive and effective, especially in the United States, where the sales volume of testosterone preparations keeps rising, doubling between 2005 and 2010 [37]. Kaufman offers a more measured standpoint, emphasizing that the finding of a severe $\mathrm{LOH}$ should be taken by clinicians as a warning of a potentially highrisk health status of their patient and should prompt them to take appropriate actions, which in the present state of the art should not include testosterone treatment [38]. In Poland, such aggressive marketing is not noticeable, the majority of preparations are not even present on the market, and testosterone use in patients with diagnosed TDS is, unfortunately, marginal. Introduction of the selective androgen receptor modulators (SARMs) to clinical practice might be of importance as it would allow to exclude the detrimental effect on the prostate and the cardiovascular system, with simultaneous benefits for the sexual functions, mood, and osteoporotic prevention [39].
Apart from the direct impact of $\mathrm{LOH}$ on the quality of life of the ageing males, its predictive value is increasingly debated: subjects with compensated or overt hypogonadism had an increased predicted risk of cardiovascular events (assessed by Progetto Cuore risk algorithm), as compared to eugonadal individuals. Accordingly, mortality related to major adverse cardiovascular events (MACEs), but not MACE incidence, was significantly higher in subjects with both compensated and overt hypogonadism as compared to eugonadal subjects. The possibility that subclinical hypogonadism could be a normal response of the hypothalamus-pituitary-testis axis to somatic illness should be considered [40].

In conclusion, in light of new population-based studies, the existence of the clinical and biochemical syndrome known as LOH has been confirmed, but its incidence appears to be notably lower than originally estimated. Regardless, a group of authors who are in direct opposition remains active. Research and recommendations of Lunnefeld postulate to initiate therapy even at a testosterone level of $15 \mathrm{nmol} / \mathrm{l}$ and presence of clinical manifestations. The general consensus is that $\mathrm{LOH}$ constitutes a predictive value for the entire well-being of a man and may identify him as a highrisk candidate for numerous diseases. However, there is no consensus on the risk-benefit index in testosterone therapy. A large interventional study, currently conducted in the USA and scheduled to end in 2015, offers hope to break the impasse.

\section{Disclosure}

Authors report no conflict of interest.

\section{References}

1. Chen CY, Lee CP, Chen Y, et al. The correlation between emotional distress and aging males' symptoms at a psychiatric outpatient clinic: sexual dysfunction as a distinguishing characteristic between andropause and anxiety/depression in aging men. Clin Interv Aging 2013; 8: 635-640.

2. Sato Y, Tanda H, Kato S, et al. Prevalence of major depressive disorder in self-referred patients in a late onset hypogonadism clinic. Int J Impot Res 2007; 19: 407-410.

3. Humeniuk E, Bojar I, Owoc A, et al. Psychosocial conditioning of depressive disorders in post-menopausal women. Ann Agric Environ Med 2011; 18: 441-445.

4. Heller CG, Myers GB. The male climacteric, its symptomatology, diagnosis and treatment. JAMA 1944; 126: 472-477.

5. Heinemann K, Saad F. Sweating attacks: key symptom in menopausal transistion only for women? Eur Urol 2003; 44: 583-587.

6. Heinemann LAJ, Thiel C, Assman A, et al. Sex differences of "climacteric symptoms" with inceraing age? A pooled analysis of cross-sectional population-based surveys. Aging Male 2000; 3: 124-131.

7. Harrison J. 'Talking about my generation': a state-of-the-art review of health information for men in the andropause. Health Info Libr J 2011; 28: 161-170.

8. Turnover JC. Androgen administration to aging men. Endocrinol Metab Clin North Am 1994; 23: 877-892.

9. Morley JE, Kaiser FE, Perry HM 3rd, et al. Longitudinal changes in testosterone, luteinizing hormone, and follicle-stimulating hormone in healthy older men. Metabolism 1997; 46: 410-413. 
10. Belchetz PE, Barth JH, Kaufman JM. Biochemical endocrinology of the hypogonadal male. Ann Clin Biochem 2010; 47: 503-515.

11. Wu CY, Yu TJ, Chen MJ. Age related testosterone level changes and male andropause syndrome. Chang Gung Med J 2000; 23: 348-353.

12. Moore C, Huebler D, Zimmermann T, et al. The Aging Males Symptoms scale (AMS) as outcome measure for treatment of androgen deficiency. Eur Urol 2004; 46: 80-87.

13. Morley JE, Charlton E, Patrick P, et al. Validation of a screening questionnaire for androgen deficiency in aging males. Metabolism 2000; 49: 1239-1242.

14. O'Donnell AB, Travison TG, Harris SS, et al. Testosterone, dehydroepiandrosterone, and physical performance in older men: results from the Massachusetts Male Aging Study. J Clin Endocrinol Metab 2006; 91: 425-431.

15. Araujo AB, Esche GR, Kupelian V, et al. Prevalence of symptomatic androgen deficiency in men. J Clin Endocrinol Metab 2007; 92: 4241-4247.

16. Huhtaniemi I. Late onset hypogonadism: current concepts and controversies of pathogenesis, diagnosis and treatment. Asian J Androl 2014; 16: 192-202.

17. Nieschlag E, Swerdloff R, Behre HM, et al. Investigation, treatment and monitoring of late-onset hypogonadism in males: ISA, ISSAM, and EAU recommendations. Int J Androl 2005; 28: 125-127.

18. Lunenfeld B, Mskhalaya G, Kalinchenko S, et al. Recommendations on the diagnosis, treatment and monitoring of late-onset hypogonadism in men - a suggested update. Aging Male 2013; 16: 143-150.

19. Lunenfeld B, Mskhalaya G, Zitzmann M, et al. Recommendations on the diagnosis, treatment and monitoring of hypogonadism in men. Aging Male 2015 [Epub ahead of print].

20. Wu FC, Tajar A, Beynon JM, et al. EMAS Group. Identification of lateonset hypogonadism in middle-aged and elderly men. $\mathrm{N}$ Engl J Med 2010; 363: 123-135

21. Huhtaniemi I, Mäkinen J, Perheentupa A, Raitakari OT. Late-onset hypogonadism in men. Experience from the Turku Male Ageing Study (TuMAS). Hormones (Athens) 2008; 7: 36-45.

22. Sansone A, Romanelli F, Gianfrilli D, et al. Endocrine evaluation of erectile dysfunction. Endocrine 2014; 46: 423-430.

23. Wylie K, Froggatt N. Late onset hypogonadism, sexuality and fertility. Hum Fertil (Camb) 2010; 13: 126-133.

24. Kapoor D, Malkin CJ, Channer KS, et al. Androgens, insulin resistance and vascular disease in men. Clin Endocrinol 2005; 63: 239-250.

25. Traish AM, Saad F, Guay A. The dark side of testosterone deficiency: II. Type 2 diabetes and insulin resistance. J Androl 2009; 30: 23-32.

26. Zitzmann M, Faber S, Nieschlag E. Association of specific symptoms and metabolic risks with serum testosterone in older men. J Clin Endocrinol Metab 2009; 91: 4335-4343.
27. Wang C, Nieschlag E, Swerdloff R, et al. ISA, ISSAM, EAU, EAA and ASA recommendations: investigation, treatment and monitoring of lateonset hypogonadism in males. Aging Male 2009; 12: 5-12.

28. Holm AC, Fredrikson MG, Theodorsson E, et al. Change in testosterone concentrations over time is a better predictor than the actual concentrations for symptoms of late onset hypogonadism. Aging Male 2011; 14: 249-256.

29. Camacho EM, Huhtaniemi IT, O'Neill TW, et al. EMAS Group. Age-associated changes in hypothalamic-pituitary-testicular function in middle-aged and older men are modified by weight change and lifestyle factors: longitudinal results from the European Male Ageing Study. Eur J Endocrinol 2013; 168: 445-455.

30. Zitzmann M, Nieschlag E. Androgen receptor gene CAG repeat length and body mass index modulate the safety of long-term intramuscular testosterone undecanoate therapy in hypogonadal men. J Clin Endocrinol Metab 2007; 92: 3844-3853.

31. Zajac A, Wilk M, Socha T, et al. Effects of growth hormone and testosterone therapy on aerobic and anaerobic fitness, body composition and lipoprotein profile in middle-aged men. Ann Agricul Environ Med 2014; 21: 156-160.

32. Wang C, Nieschlag E, Swerdloff R, et al. Investigation, treatment and monitoring of late-onset hypogonadism in males. Int J Androl 2009; 32: $1-10$

33. Singh P. Andropause: Current concepts. Indian J Endocr Metab 2013; 17 Suppl S3: S621-629.

34. Bhasin S, Cunningham GR, Hayes FJ, et al. Testosterone therapy in men with androgen deficiency syndromes: an Endocrine Society clinical practice guideline. J Clin Endocrinol Metab 2010; 95: 2536-2559.

35. Ruige JB, Ouwens DM, Kaufman JM. Beneficial and adverse effects of testosterone on the cardiovascular system in men. J Clin Endocrino Metab 2013; 98: 4300-4310.

36. Cunningham GR, Toma SM. Why is androgen replacement in males controversial? J Clin Endocrinol Metab 2011; 96: 38-52.

37. Handelsman DJ. Pharmacoepidemiology of testosterone prescribing in Australia, 1992-2010. Med J Aust 2012; 196: 642-645.

38. JM. Mortality associated to late-onset hypogonadism: reasons not to treat with testosterone? J Clin Endocrinol Metab 2014; 99: 1161-1163.

39. Coss CC, Jones A, Hancock ML, et al. Selective androgen receptor modulators for the treatment of late onset male hypogonadism. Asian Androl 2014; 16: 256-261.

40. Corona G, Maseroli E, Rastrelli G, et al. Characteristics of compensated hypogonadism in patients with sexual dysfunction. J Sex Med 2014 11: $1823-1834$. 